\title{
Comparison of child and adult vibrotactile thresholds as a function of frequency and duration
}

\author{
ROBERT D. FRISINA and GEORGE A. GESCHEIDER \\ Hamilton College, Clinton, New York 13323
}

\begin{abstract}
Psychophysical thresholds for the detection of sinusoidal vibration of the thenar eminence of the hand were determined for children and adults. The subjects in the child group were between 8 and 11 years old, and the adults were between 20 and 39 years old. Measurements were made for vibration frequencies ranging from 18 to $700 \mathrm{~Hz}$ and stimulus durations ranging from 28 to 1,000 msec. For both children and adults, thresholds were a U-shaped function of stimulus frequency. However, the exact form of the function was influenced by the age of the subjects. At frequencies above $200 \mathrm{~Hz}$, thresholds were virtually identical for children and adults, but below this frequency children were more sensitive than adults. This finding, in combination with known changes in the anatomy of the Pacinian corpuscle with age, is in agreement with a filter model of this cutaneous receptor. Furthermore, variation of stimulus duration resulted in data in agreement with the theory that two classes of mechanoreceptors, Pacinian and nonPacinian, mediate the perception of vibration.
\end{abstract}

On the basis of psychophysical data obtained in a study of the absolute threshold for vibration of the skin, Verrillo (1963) proposed a duplex model of mechanoreception. According to this model, the detection of vibration is mediated by at least two functionally distinct populations of mechanoreceptors. Pacinian corpuscles were assumed to mediate the psychophysical threshold for detecting high-frequency vibration, whereas quickly adapting non-Pacinian mechanoreceptors, perhaps Meissner corpuscles in glabrous skin and hair follicle endings in hairy skin, were assumed to determine the threshold at low frequencies.

Since Verrillo's original proposal, there has accumulated a large amount of psychophysical data (for reviews see Gescheider, 1976; Verrillo, 1968) and electrophysiological data (e.g., Mountcastle, LaMotte, \& Carli, 1972; Talbot, Darian-Smith, Kornhuber, \& Mountcastle, 1968) in support of the duplex model. Furthermore, the results of recent psychophysical studies support the applicability of the model to the perception of suprathreshold vibration (Gescheider, Verrillo, Capraro, \& Hamer, 1977; Verrillo \& Gescheider, 1975, 1977).

With but one exception, adult subjects have been used in psychophysical experiments on the detection of vibrotactile stimuli. In a recent study by Verrillo (1977), children with an average age of 10 years were more sensitive than adults to sinusoidal vibrations at most frequencies. This finding was accounted for in terms of anatomical changes that occur in Pacinian corpuscles as a function of age. In Verrillo's study, the loss of sensitivity with age appeared to be specific to Pacinian corpuscles.
In the present study, an attempt was made to extend Verrillo's finding on the vibrotactile sensitivity of children. By using a slightly wider range of stimulus frequencies, the transition point on the frequency dimension where one receptor population becomes more sensitive than the other could be precisely determined for children and adults. In addition, the amount of temporal summation, as indicated by changes in threshold as a function of stimulus duration, was compared for children and adults.

\section{EXPERIMENT 1}

The aim of the first experiment was to determine vibrotactile thresholds as a function of frequency for children and adults.

\section{Method}

Subjects. The median age of the child group, consisting of five females and two males, was 10 years with a range of 8 to 11 years. The adult group, consisting of two females and three males, had a median age of 20 years, with a range of 20 to 39 years. Prior to the experiment, all subjects were given several practice sessions in tracking their vibrotactile thresholds by the Békésy method.

Apparatus. Sinusoidal vibratory stimuli were delivered to the thenar eminence of the subject's right hand. The contactor of the vibrator was adjusted at the start of each session to make a 1.0-mm depression in the skin surface over the contactor. By using a rigid surround upon which the subject's hand rested, vibration of the skin was concentrated in the region of the contactor. There was a $1.0-\mathrm{mm}$ gap between the surround and the contactor. Vibration amplitude was measured with a calibrated accelerometer mounted on the moving element of the vibrator. All measurements were made during stimulation of the skin and were expressed in decibels re $1.0 \mu \mathrm{m}$ peak movement of the contactor into the skin. 
Sinusoidal electrical signals of variable frequency were passed through an electronic switch, amplified, passed through a recording attenuator, and applied to an Electrodyne V6 vibrator. The electronic switch was adjusted to provide a 100 -msec rise-decay time of the stimulus. In the first experiment, the stimulus was on for $1 \mathrm{sec}$ and off for $1 \mathrm{sec}$.

The subject was located in a booth that was relatively isolated from sound and vibration in the building. To mask the sound of the vibrator, the subject listened through earphones to narrowband noise centered around the frequency of the vibrotactile stimulus. The vibrator assembly was positioned on the platen of a drill press which could be precisely raised and lowered to regulate the penetration of the contractor into the subject's hand as it rested on the table above the vibrator. The contactor protruded through a hole in the table. The size of the contactor was $3.0 \mathrm{~cm}^{2}$ and was shaped to contour the surface of the skin of the thenar eminence of the hand.

Procedure. Thresholds were measured by the Békésy tracking method. The increases and decreases in intensity that resulted from the subject's threshold tracking were recorded as a function of time on the recording attenuator. Subjects tracked their thresholds for 1-2 min for each experimental condition. The voltage output of the accelerometer was measured for each threshold and converted to peak displacement of the vibrator in decibels relative to $1.0 \mu \mathrm{m}$. For each stimulus frequency, three thresholds were obtained from each adult and two from each child.

\section{Results and Discussion}

Mean thresholds as a function of frequency for adults and children are compared in Figure 1. The form of the adult curve corresponds to previously reported threshold-frequency functions obtained under comparable conditions (Gescheider, 1976; Verrillo, 1963). The function is flat up to $40 \mathrm{~Hz}$, whereupon the threshold begins to decrease at a rate of $12 \mathrm{~dB}$ per doubling of frequency until maximum sensitivity is reached at approximately $250 \mathrm{~Hz}$. Above $250 \mathrm{~Hz}$, sensitivity diminishes with further increases in frequency. In the present study, as in earlier studies, the U-shaped portion of the curve starting at $40 \mathrm{~Hz}$ is attributed to the frequency response of the Pacinian corpuscle. The close correlation between this psychophysical curve and the neural response of the Pacinian corpuscle has been established (e.g., Mountcastle et al., 1972; Talbot

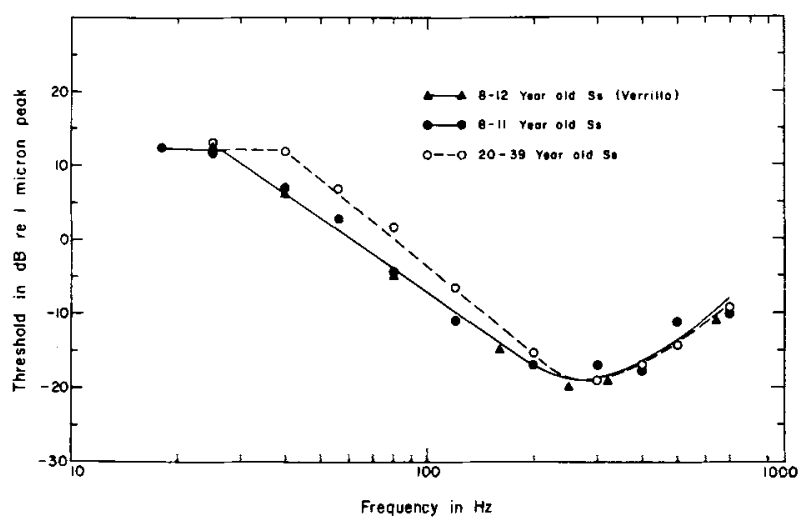

Figure 1. Vibrotactile thresholds as a function of frequency for children and adults. et al., 1968; Verrillo, 1966). The flat portion of the curve is thought to reflect the frequency response of non-Pacinian receptors (Verrillo, 1968). Although Meissner corpuscles have been identified as a mechanoreceptor in glabrous skin having a relatively flat frequency response between 20 and $40 \mathrm{~Hz}$ (Mountcastle et al., 1972; Talbot et al., 1968), there may be other mechanoreceptors in the non-Pacinian population that determine the psychophysical threshold.

For children, the discontinuity in the threshold function occurred at $25 \mathrm{~Hz}$ rather than $40 \mathrm{~Hz}$. The child curve constructed from the data of the present experiment and Verrillo's (1977) experiment is essentially flat between 18 and $25 \mathrm{~Hz}$ but decreases at a rate of $10 \mathrm{~dB}$, rather than $12 \mathrm{~dB}$, per doubling of frequency beyond $25 \mathrm{~Hz}$. The standard error of the mean for each data point was computed, and these values ranged between .57 and $1.8 \mathrm{~dB}$ with a median value of $1.0 \mathrm{~dB}$. It is noteworthy that the sensitivities of children and adults are the same for frequencies that lie within the flat portions of their curves below the points of discontinuity. Thus, the sensitivity of the population of non-Pacinian receptors that mediates the detection of low-frequency vibration did not appear to be influenced by the age of our subjects. In contrast to non-Pacinian receptors, Pacinian corpuscles appear to be affected by age. On the sloping portion of the threshold curve traditionally attributed to Pacinian corpuscles, the thresholds of children were lower than those of adults. An inference from the psychophysical data is that Pacinian corpuscles in children have a slightly broader frequency response than they do in adults.

Cauna (1965), by examining skin samples from over 200 individuals ranging in age from birth to 93 years, demonstrated that throughout life lamellae are added to Pacinian corpuscles, and consequently they become larger and distorted in shape. The lamellae surrounding the nerve fiber are built up like the layers of an onion and are separated by viscous fluid. The lamellated corpuscle surrounding the nerve fiber has been found to modify the mechanical stimulus but not to contribute to the neural excitatory process directly (Lowenstein \& Rathkamp, 1958). The corpuscle acts as a high-pass mechanical filter attenuating steady or slowly changing pressures but permitting stimulation of the nerve by rapid changes (Mendelson \& Lowenstein, 1964). Because of the mechanical filter that intervenes between mechanical disturbances on the skin and the inner core, the Pacinian corpuscle must be displaced with a critical velocity in order for the nerve fiber to be displaced. High-frequency stimuli are transmitted more efficiently than low-frequency stimuli of the same amplitude because their displacement velocities are higher. A model of the physical properties of the Pacinian corpuscles has been developed by Lowenstein 
and Skalak (1966) to account for its filtering characteristics. According to Verrillo (1977), lamellae added to the Pacinian corpuscle with age should accentuate its high-pass filtering characteristics. The psychophysical data are consistent with this hypothesis.

\section{EXPERIMENT 2}

In Experiment 2, the phenomenon of temporal summation was investigated. Because it was found, in the first experiment, that the threshold function is influenced by the age of the subjects, it was possible to make quantitative predictions of temporal summation that were different for children and adults.

The results of several studies in which adult subjects were used seem to indicate that the non-Pacinian and Pacinian systems can be distinguished on the basis of their ability to integrate stimulation over time. Verrillo (1965), using high-frequency stimulation of areas of the skin containing Pacinian corpuscles, found that the threshold for vibration decreased as a function of increases in duration up to about $1.0 \mathrm{sec}$. When the contactor is so small that only non-Pacinian receptors are stimulated at threshold (Verrillo, 1965), or when a region of the skin containing no Pacinian corpuscles is stimulated (Verrillo, 1968), no temporal summation is observed. Furthermore, when stimulus frequency is so low as to stimulate only non-Pacinian receptors, the psychophysical threshold changes little more than a decibel as the duration of the stimulus is changed (Gescheider, 1976; Gescheider et al., 1977).

In the present experiment, the effect of stimulus duration on the vibrotactile threshold was examined at three stimulus frequencies. It is predictable from the duplex model that the three chosen frequencies $(25,40$, and $200 \mathrm{~Hz})$ should result in different amounts of temporal summation. Furthermore, the amount of temporal summation was predicted to be the same for children and adults when the frequency was $200 \mathrm{~Hz}$, but for the frequencies near the point of transition from one receptor system to the other, different amounts of temporal summation were expected for children and adults.

\section{Method}

Subjects. The seven children who served in the first experiment also served as subjects in the second experiment. Six adults, ranging in age from 19 to 39 years, served as subjects. Three of the adult subjects had served in the first experiment. The three additional adult subjects were given extensive practice prior to the experiment in tracking vibrotactile thresholds by the Békésy method.

Apparatus. The apparatus was identical to that used in the first experiment, with the exception that the rise time of the stimulus was $10 \mathrm{msec}$.

Procedure. Thresholds were measured by the Békésy tracking procedure. Two thresholds were obtained from each subject at each of several stimulus durations. Stimulus duration was specified as the duration of the envelope of the signal measured at the half-power point. Data were obtained from children when stimulus frequencies were $25 \mathrm{~Hz}, 40 \mathrm{~Hz}$, and $200 \mathrm{~Hz}$ and from adults when the frequency of vibration was $40 \mathrm{~Hz}$.

\section{Results and Discussion}

The results expressed as mean threshold shifts in decibels relative to the threshold at $1,000 \mathrm{msec}$ are presented in Figure 2. The reliability of the data was indicated by relatively small intersubject variability. The standard error of the mean varied over a range of .37 to $1.0 \mathrm{~dB}$, and the median value was $.69 \mathrm{~dB}$. The threshold shift as a function of stimulus duration predicted from Zwislocki's (1960) theory of temporal summation is indicated by the curve. Zwislocki's theory accurately predicted the data obtained with children when stimulus frequency was $200 \mathrm{~Hz}$. This result is essentially identical to that reported for adults when conditions of measurement were comparable (Gescheider, 1976; Verrillo, 1965).

An assumption of the duplex model has been that with threshold functions such as those in Figure 1 a linear extrapolation of the flat portion of the curve can be used to describe the sensitivity of the nonPacinian system to higher frequencies (see Verrillo, 1968). From the curves in Figure 1, it was estimated that the threshold of the non-Pacinian system was $12 \mathrm{~dB}$ at all frequencies for both children and adults. From the detection thresholds at $200 \mathrm{~Hz}$, it can be estimated that the threshold of the Pacinian system is nearly $30 \mathrm{~dB}$ lower than that of the non-Pacinian system at this frequency. Thus there was an ample range of stimulus intensity over which temporal summation of the Pacinian system could manifest itself in changes in the psychophysical threshold.

It can be seen from Figure 1 that, as the frequency of the stimulus is reduced, the difference between the Pacinian and non-Pacinian thresholds decreases as the point of discontinuity in the curve is approached. In adults, the Pacinian and non-Pacinian systems appear to have the same thresholds at $40 \mathrm{~Hz}$.

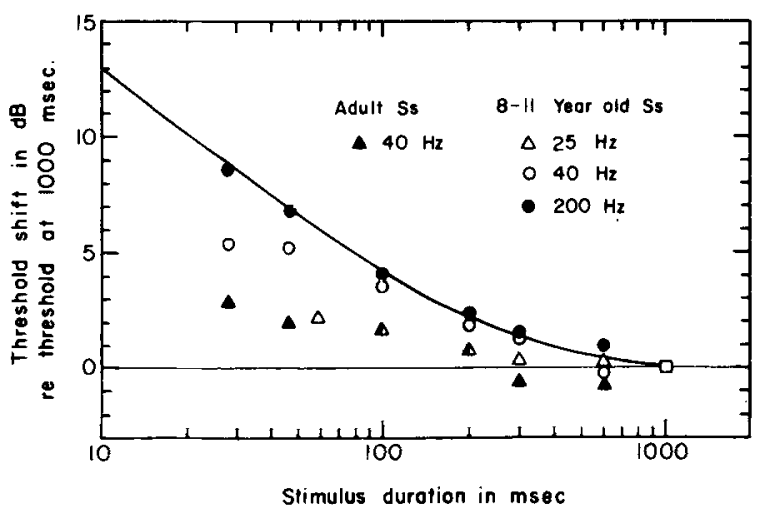

Figure 2. Shift in vibrotactile threshold as a function of stimulus duration. The curve is theoretical (Zwislocki, 1960). 
For children, on the other hand, the Pacinian system threshold is about $5.5 \mathrm{~dB}$ lower than the threshold of the non-Pacinian system. Thus, at this frequency, different temporal summation curves were expected for children and adults. The data in Figure 2 are in agreement with this expectation.

The child data follow Zwislocki's theoretical curve down to about $70 \mathrm{msec}$, at which point the threshold is elevated about $5.5 \mathrm{~dB}$. Further decreases in stimulus duration have no effect on threshold. Presumably, the temporally summating Pacinian system determines the psychophysical threshold for stimuli longer than $70 \mathrm{msec}$. However, for stimuli shorter than $70 \mathrm{msec}$ the threshold of the Pacinian system is higher than that of the non-Pacinian system, and consequently the nonsummating system determines the psychophysical threshold. Adult thresholds were only slightly affected by changes in stimulus duration, and the small threshold shifts did not follow the Zwislocki temporal summation curve.

According to the duplex model, no threshold shift should occur when the Pacinian and non-Pacinian systems have the same thresholds, because the sensitivity of the non-Pacinian is not affected by changes in stimulus duration. However, adult $40-\mathrm{Hz}$ thresholds were shifted about $2.5 \mathrm{~dB}$ when stimulus duration was less than $100 \mathrm{msec}$. One possible explanation of this result is based on the assumption that the sensitivites of the two systems were not always the same for $40-\mathrm{Hz}$ stimuli. While the data in Figure 1 suggest that when stimulus duration was $1,000 \mathrm{msec}$ the average sensitivities for the Pacinian and non-Pacinian systems were the same at this frequency for adults, it is not likely that this was true on every trial. Independent random fluctuations in the sensitivites of the two systems should result in two coincident probability distributions, and the probability of stimulus detection should be the summation of the probabilities that the sensitivity of one or the other or both systems will exceed the subject's judgment criterion. When stimulus duration is reduced, the sensitivity of the Pacinian system should be reduced and the probability of detection should eventually be determined solely by the sensitivity distribution of the non-Pacinian system. In the threshold tracking experiment, this effect would manifest itself as a compensatory elevation in stimulus intenstiy. The present analysis provides an alternative to the hypothesis that the non-Pacinian system is capable of temporal summation.

It is interesting that adults and children showed the same small temporal summation effects at 40 and $25 \mathrm{~Hz}$, respectively. In both cases, the average sensitivities of the two systems are presumed to be the same under these conditions. Temporal summation data on adults for frequencies that are below the point of discontinuity on the threshold function (where the Pacinian system is significantly less sensitive than the non-Pacinian system) indicate threshold shifts of only about $1.0 \mathrm{~dB}$ for stimulus frequencies of $30 \mathrm{~Hz}$ (Gescheider, 1976) and $25 \mathrm{~Hz}$ (Gescheider et al., 1977). It would be difficult to obtain comparable data on children because the period of 10 or $15 \mathrm{~Hz}$ vibration is so long that the manipulation of stimulus duration would be greatly restricted.

\section{REFERENCES}

Cauna, N. The effects of aging on the receptor organs of the human dermis. In W. Montagna (Ed.), Advances in biology of skin (Vol. VI) Aging. New York: Pergamon, 1965. Pp. 63-96.

Gescheider, G. A. Evidence in support of the duplex theory of mechanoreception. Sensory Processes, 1976, 1, 68-76.

Gescheider, G. A., Verrillo, R. T., Capraro, A. J., \& HAMER, R. D. Enhancement of vibrotactile sensation magnitude and predictions from the duplex model of mechanoreception. Sensory Processes, 1977, 3, 187-203.

Lowenstein, W. R., \& Rathkamp. R. The sites tor mechanoelectric conversion in a Pacinian corpuscle. Journal of General Physiology, 1958, 41, 1245-1265.

Lowenstein, W. R., \& Skalak, R. Mechanical transmission in a Pacinian corpuscle. An analysis and a theory. Journal of Physiology, 1966, 182, 346-378.

Mendelson, M., \& Lowenstein, W. R. Mechanisms of receptor adaptation. Science, 1964, 144, 554-555.

Mountcastle, V. B., LaMotte, R. H., \& Carli, G. Detection thresholds for stimuli in humans and monkeys: Comparison with threshold events in mechanoreceptive afferents nerve fibers innervating the monkey hand. Journal of Neurophysiology, $1972,35,122-136$.

Talbot, W. H., Darian-Smith, I., Kornhuber, H. H., \& Mountcastle, V.B. The sense of flutter-vibration: Comparison of the human capacity with response patterns of mechanoreception afferents from the monkey hand. Journal of Neurophysiology, 1968, 31, 301-334.

VERRILLO, R. T. Effect of contactor area on the vibrotactile threshold. Journal of the Acoustical Society of America, 1963, 35, 1962-1966.

VERRILLO, R. T. Temporal summation in vibrotactile sensitivity. Journal of the Acoustical Society of A merica, 1965, 37, 843-846.

VERRILlo, R. T. Vibrotactile sensitivity and the frequency response of Pacinian corpuscles. Psychonomic Science, 1966, 4. 135-136.

VERRILLo, R. T. A duplex mechanism of mechanoreception. In D. R. Kenshalo (Ed.), The skin senses. Springfield, Ill: Thomas, 1968. Pp. 139-159.

Verrillo, R. T. Comparison of child and adult vibrotactile thresholds. Psychonomic Bulletin, 1977, 9, 197-200.

Verrillo, R. T., \& Gescheider, G. A. Enhancement and summation in the perception of two successive vibrotactile stimuli. Perception \& Psychophysics, 1975, 18, 128-136.

Verrillo, R. T., \& Gescheider, G. A. Effect of double ipsilateral stimulation on vibrotactile sensation magnitude. Sensory Processes, 1977, 1, 127-137.

ZWISLOCKI. J. Theory of temporal auditory summation. Journal of the Acoustical Society of America, 1960, 32, 1046-1060.

(Received for publication November 12, 1976; revision accepted May 6, 1977.) 